\title{
Application and Popularization of Energy Formula
}

\author{
Dan Liu \\ Sichuan Normal University, Chengdu, China \\ Email: 576672568@qq.com
}

How to cite this paper: Liu, D. (2021) Application and Popularization of Energy Formula. Journal of Applied Mathematics and Physics, 9, 370-378.

https://doi.org/10.4236/jamp.2021.92025

Received: January 9, 2021

Accepted: February 23, 2021

Published: February 26, 2021

Copyright (C) 2021 by author(s) and Scientific Research Publishing Inc. This work is licensed under the Creative Commons Attribution International License (CC BY 4.0).

http://creativecommons.org/licenses/by/4.0/

\begin{abstract}
In physics, there are two main energy formulas. One is kinetic energy formula and the another is Einstein equation. But kinetic energy formula can only calculate low speed motion. Einstein equation can only calculate light speed motion. The two formulas are not unified. We hope to get a unified formula. But it didn't work. According to the principle of Lorentz contraction, we generalize the contraction of length to the contraction of mass, and obtain a unified energy formula. This is the generalized Einstein equation and the new Einstein kinetic energy formula.
\end{abstract}

\section{Keywords}

Kinetic Energy Formula, Energy and Mass Conversion, Lorentz Contraction Principle, Generalized Einstein Equation

\section{Introduction}

From mechanical energy, chemical energy, electric energy to nuclear energy has become the main subject of physics research.

The great energy we have now is the polymerization of hydrogen into helium. However, there is no unified formula for the calculation of energy.

In physics, there are two main energy formulas. One is kinetic energy formula. The another is Einstein equation. But kinetic energy formula can only calculate low speed motion. Einstein equation can only calculate light speed motion. The two formulas are not unified. We hope to get a unified formula. But it didn't work. It's difficult. Now let's look at a formula:

$$
w=\frac{m v^{2}}{2}
$$

Here (1.1) is called kinetic energy formula. $w$ is kinetic energy. $m$ is the mass. 
$V$ is the velocity of the object.

\section{For example,}

let $m=2 \mathrm{~kg}, V=100 \mathrm{M} / \mathrm{s}$, calculated by (1.1)

$$
w=2 \frac{100^{2}}{2}=10000
$$

This is the kinetic energy of an object under the action of an external force. There is motion mass. When the object moves at low speed, the motion mass is very small and can be ignored. Therefore, this formula is suitable for energy calculation at low speed. But it is not suitable for energy calculation of large speed, especially the energy calculation of light speed.

In 1905, Einstein published his special theory of relativity and proposed an energy formula [1]:

$$
E=m_{0} c^{2},
$$

Here (1.2) is called Einstein equation. $E$ is energy. $m_{0}$ is the static mass. The speed of light $c=299,792,457.4 \pm 0.1 \mathrm{M} / \mathrm{s}$.

Obviously, the formula is not suitable for (1.1). Can there be a formula suitable for (1.1) and (1.2)? Einstein improved (1.1)

Let the rest mass $m_{0}$, the motion velocity $v$, the motion mass $m_{b}$

$$
m_{v}=\frac{m_{0}}{\sqrt{1-v^{2} / c^{2}}}
$$

$\sqrt{1-v^{2} / c^{2}}$ It's called Lorentz contraction factor. It is not difficult to confirm that $m_{v}>m_{0}$, so as to obtain the changed mass $\Delta m$

$$
\begin{gathered}
\Delta m=m_{v}-m_{0}=\frac{m_{0}}{\sqrt{1-v^{2} / c^{2}}}-m_{0}, \\
\Delta m=m_{0}\left(\frac{1}{\sqrt{1-v^{2} / c^{2}}}-1\right),
\end{gathered}
$$

Substituting (1.2) $E=\Delta m c^{2}$, the kinetic energy formula is obtained

$$
\begin{gathered}
E=\Delta m c^{2}=m_{0}\left(\frac{1}{\sqrt{1-v^{2} / c^{2}}}-1\right) c^{2}, \\
E=m_{0}\left(\frac{1}{\sqrt{1-v^{2} / c^{2}}}-1\right) c^{2},
\end{gathered}
$$

Here (a) is Einstein's improved kinetic energy formula. Let's look at the scope of the kinetic energy formula. Let $v \ll c$, we get

$$
\frac{1}{\sqrt{1-v^{2} / c^{2}}}=1+\frac{v^{2}}{2 c^{2}}+\cdots,
$$

Take main item $1+\frac{v^{2}}{2 c^{2}}$, by (a) get (1.1) 


$$
E=m_{0}\left(1+\frac{v^{2}}{2 c^{2}}-1\right) c^{2}=\frac{1}{2} m_{0} v^{2},
$$

The kinetic energy formula (a) is suitable for (1.1). Let $v=c$, by (a) get

$$
E=m_{0}\left(\frac{1}{\sqrt{1-c^{2} / c^{2}}}-1\right) c^{2},
$$

It tends to infinity. The kinetic energy formula (a) is not suitable for (1.2). It seems that it is difficult to obtain the formula which is suitable for (1.1) and (1.2).

Let's look at the Journal of Shaanxi Normal University (volume 03.31, April, 3857.53)_ 03) Professor Chen Junfu's research on kinetic energy formula.

Variable acceleration motion and new kinetic energy formula

Let the initial acceleration be $a_{0}$ and the final acceleration be $a_{v}$, then the average acceleration can be obtained.

Let the initial acceleration be $a_{0}$ and the final acceleration be $a_{v}$, then the average acceleration can be obtained

$$
\bar{a}=\frac{a_{0}+a_{v}}{2},
$$

Can get $v^{2}=2 \bar{a} s=\left(a_{0}+a_{v}\right) s$,

Substituting $a_{v}=a_{0} \frac{c-v}{c}$

Get

$$
v^{2}=\left(a_{0}+a_{0} \frac{c-v}{c}\right) s=a_{0} \frac{2 c-v}{c} s,
$$

From the above $s=\frac{c}{a_{0}(2 c-v)} v^{2}$,

Substituting

$$
E=m_{0} a_{0} \frac{c}{a_{0}(2 c-v)} v^{2},
$$

Namely

$$
E=\frac{c}{2 c-v} m_{0} v^{2}
$$

Here $(c)$ is the new kinetic energy formula, where $E$ is kinetic energy. $m_{0}$ is the static mass. $v$ is the speed of motion.

Let's look at the scope of application of formula $(c)$. We get

$$
E=\frac{c}{2 c-v} m_{0} v^{2}=\frac{1}{2-v / c} m_{0} v^{2},
$$

Let $v \ll c, \quad v / c \approx 0$, we get $(1.1)$

$$
E=\frac{1}{2} m_{0} v^{2},
$$

Let $v=\mathcal{c}$, we get $(1.2)$ 


$$
E=\frac{c}{2 c-c} m_{0} c^{2}=m_{0} c^{2}
$$

Obviously, formula $(c)$ is suitable for (1.1) and (1.2).

However, there is a problem in the reasoning of formula $(c) . \frac{c}{2 c-v}$ How is it obtained? Unclear.

Besides, $E=m_{0} a_{0} s$, it's not right. $a_{0}$ should be the average acceleration. It should be

$$
E=m_{0} \bar{a} s
$$

From the front $v^{2}=2 \bar{a} s$ get $\bar{a} s=\frac{v^{2}}{2}$, From this we get

$$
E=m_{0} \bar{a} s=\frac{m_{0} v^{2}}{2},
$$

It's still formula (1.1). It's not a new kinetic energy formula.

In this paper, we use Lorentz contraction principle [2] to generalize Einstein's equation, and obtain generalized Einstein's equation and new Einstein's kinetic energy formula.

\section{Lorentz Contraction Principle}

In 1892, the physicist Lorentz, based on the practical research of Michelson and Murray, proposed that when an object moves, it shrinks in the direction of motion. This is the famous Lorentz contraction.

Let the length before the object moves be $L_{0}$, the length when the object moves be $L_{v}$, and the velocity be $v$ get [1] [2] [3] [4]:

$$
L_{v}=L_{0} \sqrt{1-\frac{v^{2}}{c^{2}}}
$$

Here (2.1) is called Lorentz contraction principle. It's called Lorentz contraction factor. $L_{V}$ is called motion length. The speed of light $c=299,792,457.4 \pm 0.1$ $\mathrm{M} / \mathrm{s}$.

\section{for example,}

Let $L=2 \mathrm{~m}, V=100 \mathrm{M} / \mathrm{s}$, calculated by $(2.1)$

$$
L_{v}=2 \sqrt{1-\frac{100^{2}}{299792457.4^{2}}}=1.999999999999888734993949266929,
$$

The greater the speed, the smaller the movement length,

Confirmed by (2.1) $L_{0}>L_{v}$, From (2.1) we get the length of the change $\Delta L$

$$
\Delta L=L_{0}-L_{v}=L_{0}-L_{0} \sqrt{1-\frac{v^{2}}{c^{2}}}
$$

By (2.2) get

$$
\Delta L=L_{0}\left(1-\sqrt{1-\frac{v^{2}}{c^{2}}}\right)
$$


Represents the length of an object as it moves. Let's look at the length of motion of an object moving at the speed of light.

Let $v=\mathcal{c}$, and the length of change is obtained from (2.2)

$$
\Delta L=L_{0}\left(1-\sqrt{1-\frac{c^{2}}{c^{2}}}\right)=L_{0}
$$

Represents the maximum length of change when an object moves at the speed of light.

\section{Mass Shrinkage Formula}

We generalize Lorentz length contraction to mass contraction. The length of motion $L_{V}$ is extended to motion Quality $m_{v}$.

Let $m_{0}$ be the mass before the object moves, $m_{V}$ be the mass when the object moves, $v$ be the velocity, get

$$
m_{v}=m_{0} \sqrt{1-\frac{v^{2}}{c^{2}}}
$$

Here (3.1) is called motion mass, represents the mass of an object in motion. It was confirmed by (3.1) that $m_{0}>m_{v}$,

Let the variable mass $\Delta m$ be obtained from (3.1)

$$
\begin{gathered}
\Delta m=m_{0}-m_{v}=m_{0}-m_{0} \sqrt{1-\frac{v^{2}}{c^{2}}}, \\
\Delta m=m_{0}\left(1-\sqrt{1-\frac{v^{2}}{c^{2}}}\right),
\end{gathered}
$$

Here (3.2) represents the variable mass of an object in motion.

For example,

Let $m_{0}=2 \mathrm{~kg}, v=100 \mathrm{M} / \mathrm{s}$, calculated by (3.2)

$$
\Delta m=2\left(1-\sqrt{1-\frac{100^{2}}{299792457.4^{2}}}\right)=0.00000000000011126500605073307,
$$

The greater the speed, the greater the change of mass. Let's look at the change of mass when an object moves at the speed of light.

Let $v=c$, and the variable mass is obtained from (3.2)

$$
\Delta m=m_{0}\left(1-\sqrt{1-\frac{c^{2}}{c^{2}}}\right)=m_{0}
$$

Represents the maximum mass that changes when an object moves at the speed of light. According to (1.2), we generalize Einstein's equation [5], transform mass into energy, and get the generalized Einstein's equation [6].

\section{Generalized Einstein Equation}

We can get from (1.2) and (3.2) [7] 


$$
E=\Delta m c^{2}
$$

From (3.2) and (4.1), we can get [8]:

$$
E=m_{0}\left(1-\sqrt{1-\frac{v^{2}}{c^{2}}}\right) c^{2},
$$

Here (4.2) is called generalized Einstein equation.

$E$ is the energy released. $m_{0}$ is the mass of the object before it moves. The speed of light $c=299,792,457.4 \pm 0.1 \mathrm{M} / \mathrm{s}$.

\section{For example,}

Let $m_{0}=2 \mathrm{~kg}, v=100 \mathrm{M} / \mathrm{s}$, calculated by $(4.2)$

$$
E=2\left(1-\sqrt{1-\frac{100^{2}}{299792457.4^{2}}}\right) c^{2}=10000.000000000278163102077068 \text {, }
$$

The value is the same as the kinetic energy formula in (1.1). Let's look at the scope of application. Let $v \ll c$, according to the previous

$$
\frac{1}{\sqrt{1-v^{2} / c^{2}}}=1+\frac{v^{2}}{2 c^{2}}=\frac{2 c^{2}+v^{2}}{2 c^{2}}
$$

Get

$$
\sqrt{1-v^{2} / c^{2}}=\frac{2 c^{2}}{2 c^{2}+v^{2}}
$$

From this we get

$$
1-\sqrt{1-v^{2} / c^{2}}=1-\frac{2 c^{2}}{2 c^{2}+v^{2}}=\frac{v^{2}}{2 c^{2}+v^{2}},
$$

Can get

$$
1-\sqrt{1-v^{2} / c^{2}}=\frac{v^{2}}{2 c^{2}+v^{2}}
$$

Substituting (4.2)

$$
E=m_{0} \frac{v^{2}}{2 c^{2}+v^{2}} c^{2}=m_{0} \frac{v^{2}}{2+v^{2} / c^{2}},
$$

$v / c \approx 0$, get

$$
E=\frac{m_{0} v^{2}}{2}
$$

The formula (4.2) is suitable for (1.1). Let $V=c$, which can be obtained from $(4.2)$

$$
E=m_{0} c^{2}
$$

This is formula (1.2). The generalized Einstein equation is suitable for both (1.1) and (1.2).

Let velocity $v$, mass $m_{0}=1, E(a)$ denote formula (a), $E(s)$ denote formula (4.2), Partial calculation: 


$\begin{array}{llll} & E(a)=m_{0}\left(\frac{1}{\sqrt{1-v^{2} / c^{2}}}-1\right) c^{2}, & E(s)=m_{0}\left(1-\sqrt{1-\frac{v^{2}}{c^{2}}}\right) c^{2}, \\ v & E(a) & E(s) & E(a) / E(s) \\ 10000 & 50000000 & 50000000 & 1 \\ 200000 & 20000006675 & 20000002225 & 1.0000002 \\ 65000000 & 2190023689283367 & 2137928152810761 & 1.0243672 \\ 299570000 & 2243549820812118646 & 86413821733172904 & 25.962858\end{array}$

The value of formula $E(a)$ is very close to that of formula $E(s)$. However, when $v$ approaches the speed of light, the deviation of $E(a)$ is obvious.

Through the above discussion, it is not difficult to find that the key to the argument is $\Delta m$. Thus, a new Einstein kinetic energy formula is obtained.

\section{Einstein's Kinetic Energy Formula}

Previously, we discussed the traditional kinetic energy formula (1.1)

$$
w=\frac{m_{0} v^{2}}{2},
$$

When an object is in motion, its mass changes $\Delta m$, The total mass was obtained

$$
m=m_{0}+\Delta m,
$$

Total energy

$$
W=\frac{m v^{2}}{2}=\frac{m_{0}+\Delta m}{2} v^{2},
$$

Namely

$$
W=\frac{m_{0} v^{2}}{2}+\frac{\Delta m v^{2}}{2},
$$

By (3.2) get

$$
\Delta m=m_{0}\left(1-\sqrt{1-\frac{v^{2}}{c^{2}}}\right),
$$

We can get

$$
\frac{\Delta m v^{2}}{2}=\frac{m_{0} v^{2}}{2}\left(1-\sqrt{1-\frac{v^{2}}{c^{2}}}\right)
$$

By (5.1) get

$$
W=\frac{m_{0} v^{2}}{2}+\frac{m_{0} v^{2}}{2}\left(1-\sqrt{1-\frac{v^{2}}{c^{2}}}\right),
$$

We can get

$$
W=\frac{m_{0} v^{2}}{2}+\frac{m_{0} v^{2}}{2}\left(1-\sqrt{1-\frac{v^{2}}{c^{2}}}\right)=\frac{m_{0} v^{2}}{2}\left(2-\sqrt{1-\frac{v^{2}}{c^{2}}}\right),
$$


The results are as follows

$$
W=\frac{m_{0} v^{2}}{2}\left(2-\sqrt{1-\frac{v^{2}}{c^{2}}}\right),
$$

Here (5.2) is called Einstein's kinetic energy formula.

Let $v \ll c, v / c \approx 0$, and can be ignored

$$
2-\sqrt{1-\frac{v^{2}}{c^{2}}}=1
$$

By (5.2) can get

$$
W=\frac{m_{0} v^{2}}{2},
$$

Formula (5.2) is suitable for (1.1).

Let $v=c$ and $v / c=1$, we get

$$
2-\sqrt{1-\frac{c^{2}}{c^{2}}}=2
$$

By (5.2) get

$$
W=m_{0} c^{2},
$$

The formula (5.2) is suitable for (1.2).

In this way, formula (5.2) is suitable from low speed to light speed.

Let velocity $V$, mass $m_{0}=1, E(W)$ denote formula (5.2), $E(s)$ denote formula (4.2),

Partial calculation:

$$
\begin{array}{llll} 
& E(W)=\frac{1}{2} m_{0}\left(2-\sqrt{1-\frac{v^{2}}{c^{2}}}\right) v^{2}, E(s)=m_{0}\left(1-\sqrt{1-\frac{v^{2}}{c^{2}}}\right) c^{2}, \\
v & E(W) & E(s) & E(W) / E(s) \\
10000 & 50000000 & 50000000 & 1 \\
200000 & 20000004451 & 20000002225 & 1.00000011 \\
65000000 & 2162751429396361 & 2137928152810761 & 1.0116109 \\
299570000 & 88013904766446535 & 86413821733172904 & 1.0185165
\end{array}
$$

The value of formula $E(W)$ is very close to that of formula $E(s)$.

\section{Conclusions}

In this paper, according to Lorentz contraction principle and Einstein equation, we get

$$
\begin{gathered}
E=k_{c} m_{0} c^{2}, \\
k_{c}=1-\sqrt{1-\frac{v^{2}}{c^{2}}},
\end{gathered}
$$

Here (6.1) is the generalized Einstein equation. It means that the mass of an object shrinks and releases energy. Also 


$$
\begin{gathered}
W=\frac{1}{2} k_{v} m_{0} v^{2}, \\
k_{v}=2-\sqrt{1-\frac{v^{2}}{c^{2}}},
\end{gathered}
$$

Here (6.2) is Einstein's kinetic energy formula. It means that the mass of an object expands and absorbs energy.

The generalized Einstein equation and Einstein's kinetic energy formula are all correct.

The above energy formula is based on the theory of mass and energy conversion. According to this theory, the wave particle image can be interpreted. Particle is mass. Waves are energy. The conversion of mass into energy is a wave. When energy is converted into mass, it is called a particle.

Light is a wave of mass and energy. When mass and energy are transformed, particles contract in the direction of motion and release energy. Then it absorbs the energy and the particles expand in the direction of motion. Particles move through contraction and expansion.

It can also explain the speed limit of light. The mass of the particle shrinks to the minimum, the energy is the maximum, and the speed is the maximum. The mass of the particle shrinks to the limit, the energy is the limit, and the speed is the limit. This speed limit is the speed of light.

The theory of mutual transformation between mass and energy can be regarded as the basis of relativity. This theory is correct.

\section{Conflicts of Interest}

The author declares no conflicts of interest regarding the publication of this paper.

\section{References}

[1] Einstein (1964) Special and General Theory of Relativity (Yang, R.Y., Trans.). Shanghai Science Press, Shanghai.

[2] Chen, J.F. and Chen, J.C. (1998) Exploration of Some Basic Problems in Physics. Shaanxi Science Press, Xi'an.

[3] Boscarino, G. (2016) Tradizioni di pensiero. La tradizione filosofica italica della scienza. Aracne, Roma.

[4] Poincaré, H. (1963) Mathematics and Science: Last Essays. Dover, New York.

[5] Feynman, R.F., Leighton, R.B. and Sands, M. (1966) The Feynman Lectures on Physics. Vol. III, 19-1, Addison-Wesley, Reading. https://doi.org/10.1063/1.3047826

[6] Newton, I. (1965) Principi matematici di filosofia naturale. UTET, Prefazione dell'autore, Torino, 1686.

[7] Democrito (1970) Raccolta dei frammenti. Bombiani, Milano.

[8] Daviau, C. (2012) Double Space-Time and More. JePublie, Pouillé-les-coteaux. 